\title{
Moderate to severe depressive symptoms and rehabilitation outcome in older adults with hip fracture
}

\author{
Sara Morghen ${ }^{1,2}$, Giuseppe Bellelli ${ }^{1,2}$, Sara Manuele ${ }^{3}$, Fabio Guerini ${ }^{1,2}$, \\ Giovanni B Frisoni ${ }^{4}$ and Marco Trabucchi ${ }^{1,5}$ \\ ${ }^{1}$ Geriatric Research Group, Brescia, Italy \\ ${ }^{2}$ Department of Rehabilitation and Aged Care Unit, Ancelle della Carità Hospital, Cremona, Italy \\ ${ }^{3}$ Department of Internal Medicine and Geriatrics, Catania University, Italy \\ ${ }^{4}$ LENITEM - Laboratory of Epidemiology Neuroimaging and Telemedicine, IRCCS San Giovanni di Dio FBF, Brescia, Italy \\ ${ }^{5}$ Tor Vergata University, Rome, Italy \\ Correspondence to: G. Bellelli, MD, E-mail: giuseppebellelli@libero.it
}

Objective: To evaluate if depressive symptoms affect recovery of walking ability and 1-year institutionalization or mortality in older adults who underwent post-hip fracture (HF) surgery rehabilitation.

Methods: Depressive symptoms were assessed on admission using the 15-item Geriatric Depression Scale (GDS), with scores $\geq 10 / 15$ indicating moderate to severe depressive symptoms. Multidimensional assessment included Mini Mental State Examination, Charlson Comorbidity Index, Body Mass Index, albumin serum levels, number of drugs, antidepressants and Barthel Index (BI) on admission and at discharge. Walking ability was evaluated using the BI walking sub-item referred to 1 month before HF, on admission, and at discharge. Patients scoring $\leq 3 / 15$ BI walking sub-item on admission (i.e. those fully dependent or requiring major supervision in walking) were included. Walking independence at discharge was defined as a score $\geq 12 / 15$ at the BI walking sub-item.

Results: In multivariate analyses, after adjustment for covariates and potential confounders, patients with moderate to severe depressive symptoms were more likely to fail walking independence at discharge (odds ratio, $\mathrm{OR}=3.2 ; 95 \% \mathrm{CI}=1.3$ to $7.8 ; p=0.010$ ) and to be institutionalized or died at 1 year $(\mathrm{OR}=3.6,95 \% \mathrm{CI}=1.4$ to $9.1, p=0.007)$. In further analyses, the failure to recover walking independence at discharge partly mediates the relationship between moderate to severe depressive symptoms and 1-year adverse events.

Conclusions: Moderate to severe depressive symptoms affect the recovery of walking independence after HF rehabilitation and are associated with severe adverse outcomes at 1 year. Copyright (C) 2010 John Wiley \& Sons, Ltd.

Key words: depression; hip fracture; functional recovery; mortality; rehabilitation

History: Received 11 May 2010; Accepted 20 September 2010; Published online 9 November 2010 in Wiley Online Library (wileyonlinelibrary.com)

DOI: $10.1002 / g p s .2651$

\section{Introduction}

Hip fracture (HF) is one of the main causes of hospital admission among the elderly population (Crotty et al., 2009). In Italy, the incidence rate of HF is about 1.4 fracture/1000 inhabitants/year, and ranges from 6.5 to $7.5 / 1000$ individuals aged over 65 , representing one of the most important causes of mortality $(20 \%$ in the first 6 months after fracture) and disability among older people (33\% not returning to the pre-morbid physical functioning) (Laforgia et al., 2006)). A study by de Luise et al. (2008) found that HF increased 1-year mortality more than 3 -fold.

The impact of depressive symptoms in post-HF surgery rehabilitation has been repeatedly evaluated with regard to patients' functional recovery at discharge and other adverse outcomes. However, the literature reports conflicting findings and a univocal position is yet to be established. In fact, while some studies reported that depressive symptoms can complicate the course of 
rehabilitation (Cullum et al., 2008) and affect functional outcomes at discharge (Lenze et al., 2004; Hershkovitz et al., 2007), others failed to find any significant association (Lieberman et al., 2006; Lenze et al., 2007; Arinzon et al., 2009).

In the attempt to clarify this issue, we undertook the present study that assessed, in a population of postsurgery HF patients, the relationship between depressive symptoms, recovery of walking independence at discharge from rehabilitation and occurrence of adverse events (mortality or institutionalization) at 1 year. A possible mediation effect of failing the recovery of walking independence between depressive symptoms and 1-year adverse events was also evaluated. Depressive symptoms were assessed at different levels of severity in order to evaluate whether there is a gradient effect among absent, mild and moderate to severe depressive symptoms and our selected outcome measures.

\section{Methods}

\section{Participants}

The study sample was taken from 4788 first admissions aged $\geq 65$ year of our 60-beds Department of Rehabilitation and Aged Care Unit from 1st January 2002 to 1st June 2006. Of these patients, 423 were admitted for post-HF surgery rehabilitation and were, therefore, eligible for this study. Exclusion criteria were as follows:

(1) Length of stay in the orthopaedic ward before admission longer than 1 week,

(2) Written order not to ambulate after HF surgery,

(3) Pathological or multiple fractures and/or other illnesses reducing life expectancy to less than 6 months,

(4) Poor reliability of self reported depressive symptoms (score $\leq 15 / 30$ on Mini Mental State Examination-MMSE) (Yesavage et al., 1983; Charlson et al., 1987; Almeida and Almeida, 1999; Bellelli et al., 2008) and

(5) Ability to walk independently or with minor assistance on admission (i.e. score $>3 / 15$ on BI walking sub-item) in order to reduce inter-patient variability at baseline.

\section{Measures}

Baseline data on demographic characteristics (age, gender and living arrangement), health, nutritional and cognitive status were obtained on admission from the best available sources, including medical records, interviews with health care professionals or family members and clinical examinations. The global assessment of health and nutritional status were assessed with the Charlson comorbidity score, a measure of comorbidity which is a strong predictor of mortality (Charlson et al., 1987), the number of drugs, the Body Mass Index (BMI), a biometric measurement expressed as the ratio between a person's weight and height and the examination of albumin serum levels. Additionally, the use of antidepressants was recorded both on admission and at discharge. Cognitive status was evaluated with the MMSE (Folstein et al., 1975).

Functional status was assessed with the modified Barthel Index (BI) (Shah et al., 1989), a tool that evaluates the degree of independence on 10 activities of daily living. Information about functional status on admission and at discharge were collected based on nurses' evaluations. The degree of walking dependence was assessed using the BI walking sub-item. A score of 0 denoted complete inability to walk independently, a score of 3 denoted necessity of major assistance at walking, a score of 8 indicated need of minor assistance to reach and use walking aids, a score of 12 described an overall condition of independence at walking for limited distances $(50 \mathrm{~m})$, while a score of 15 denoted full autonomy. The presence of depressive symptoms was assessed on admission using the 15-items Geriatric Depression Scale (GDS) (Yesavage et al., 1983; Bellelli et al., 2008). The GDS is a commonly used screening tool in geriatric settings, with higher scores indicating worse affective status. A GDS score between 0 and 5 denoted absence of depressive symptoms, scores between 6 and 9 indicated mild depressive symptoms, while scores between 10 and 15 indicated moderate to severe depressive symptoms (Brink et al., 1982).

\section{Rehabilitation training}

All subjects received an intensive physical therapy rehabilitation two times per day ( 6 day per week for all rehabilitation stay). Each session of physical therapy started with a 15-min warm-up and ended with 10-min cool-down phase.

The rehabilitation training included strengthening exercises, transfer, postural and gait training and adaptive equipment training, according to physical therapist evaluation of individual needs. At the beginning of each rehabilitation session the physiotherapist explained the nature, goal and type of the exercise. Strengthening exercises were initially used in all patients with gait 
disorders. Generally, each session consisted of a 40-min phase including leg extension, leg curl, step-up, upright row, abductions, adductions and pelvic tilt. When patients required to rest, a brief interruption (no longer than $1 \mathrm{~min}$ ) was allowed. The physiotherapists recorded the time spent in each rehabilitation session (minutes per day) and, at discharge, a sum was computed.

\section{Outcome measures}

Walking independence at discharge was defined as a score $\geq 12 / 15$ at the BI walking sub-item.

Adverse outcome at 1 year included institutionalization or death. The 1-year follow-up was conducted by structured phone interviews with proxies in order to assess vital status and living arrangement.

We obtained informed consent (or proxy consent for patients with cognitive impairment) from all patients during their rehabilitation stay. The protocol was approved by the Ethics Committee of Gerontological Sciences of the Geriatric Research Group, Italy.

\section{Statistical analysis}

All analyses were performed using the 11.0 version of SPSS package (Statistical Package for Social Sciences). The $T$ - or Mann-Whitney $U$-tests were used to examine differences of demographic, clinical, cognitive and functional characteristics between groups. Depressive symptoms were treated as categorical variables with normal score (GDS $=0-5$ ) as the reference group, scores between 6 and 9 indicating mild depressive symptoms and scores higher than 10 indicating moderate to severe depressive symptoms. In order to determine the variables predicting walking dependence at discharge and adverse outcomes at 1 year, both bivariate and multivariate logistic regressions were tested. Significance $\leq 0.20$ at the bivariate analysis ( $T$-test or Mann-Whitney $U$-test) was used to include in the multivariate regressions the variables identified as possible predictors. In order to test whether a possible mediation effect among our predictor of interest (i.e. depressive symptoms) and the two outcome measures (i.e. walking recovery and 1-year adverse outcomes), we first performed bivariate correlations among those variables, and then a standard series of multivariate regression analyses. The Sobel's statistic test was calculated with both the one-tailed and two-tailed probability values, according to a standardized method.

\section{Results}

Of the 423 patients admitted with HF during the study period, 61 scored $\leq 15 / 30$ at MMSE, 13 scored $>3 / 15$ at BI walking sub-item on admission, 10 remained in the orthopaedic ward longer than 1 week before being admitted to rehabilitation, five had a terminal illness, nine multiple or pathological fractures and eight had a written order not to ambulate. Moreover, 37 patients were lost at the 1-year follow-up. The final sample included 280 subjects. Table 1 summarizes baseline characteristics of the whole population, stratified by walking ability at discharge and adverse events at 1 year. Of all patients, other than nearly two-thirds $(n=192$, $68.6 \%$ ) were able to walk independently at discharge, while the remaining patients were not. The two groups (able and unable) were comparable by gender, nutritional status, walking ability on admission and length of stay. Patients who did not recover walking independence at discharge were older, more likely not to live alone and more dependent in walking before fracture, had greater cognitive impairment, more frequently depressive symptoms (as indirectly confirmed also by the use of antidepressants) and had poorer health status (greater comorbidity, lower albumin serum levels and higher number of drugs) on admission than their counterpart. At the 1-year follow-up, 50 patients (17.9\%) have died or were institutionalized, while $230(82.1 \%)$ have not. Again, groups were similar for gender, living arrangement, nutritional and clinical status, number of drugs taken, walking ability on admission and length of stay, but patients with adverse events at 1 year were averagely older and more cognitively impaired, had more frequently depressive symptoms, worse walking ability before fracture and were less likely to have recovered walking independence during rehabilitation stay than patients in the other group. There were no differences on the length of stay and time spent in rehabilitation according to the severity of depressive symptoms. Specifically, patients without depressive symptoms had a length of stay of $28.17 \pm 10.7$ days vs. $27.18 \pm 7.2$ for patients with mild depressive symptoms, and $29.27 \pm 11.2$ for patients with moderate to severe depressive symptoms. There were no differences among groups at the Analysis Of Variance Test (ANOVA) $(p=0.50)$, Although patients with no depressive symptoms had the more intensive rehabilitation regimen $(58.0 \pm 15.3 \mathrm{~min}$ per day), followed by those with mild depressive symptoms $(56.4 \pm 16.2 \mathrm{~min}$ per day), then those with moderate to severe depressive symptoms $(51.3 \pm 13.4 \mathrm{~min}$ per day) in a decreasing order, the differences among these three groups were not statistically significant (data not shown in the Tables). 


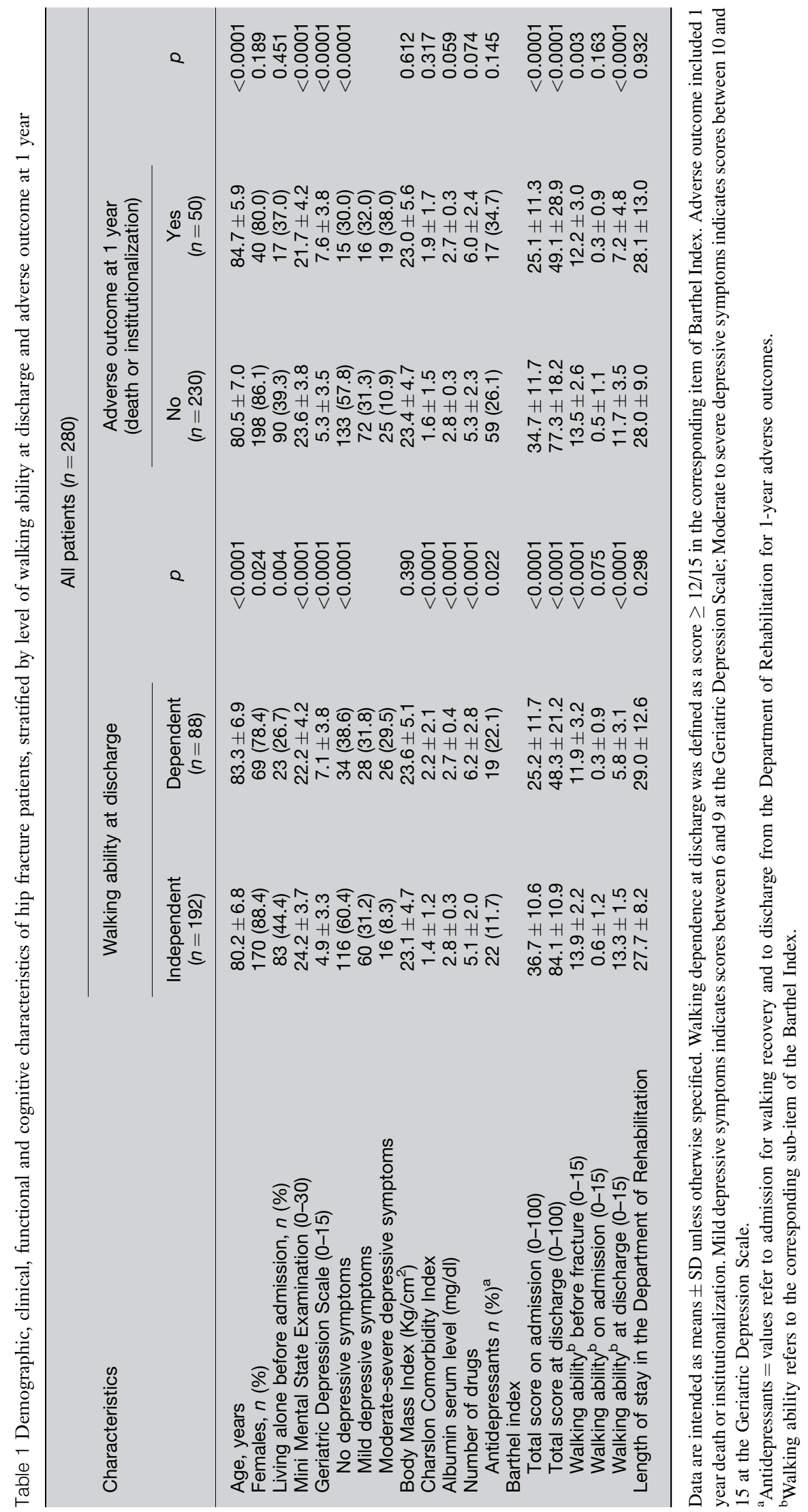


Table 2 Independent predictors of failure to recover walking independence at discharge in 280 patients consecutively admitted to a Department of Rehabilitation and Aged Care Unit after hip fracture surgery in unadjusted and adjusted multiple logistic regression models

\begin{tabular}{|c|c|c|c|c|c|c|c|c|}
\hline & \multicolumn{8}{|c|}{ Failure to recover walking independence at discharge } \\
\hline & \multicolumn{4}{|c|}{ Unadjusted } & \multicolumn{4}{|c|}{ Adjusted } \\
\hline & OR & Wald $\chi^{2}$ & $95 \% \mathrm{Cl}$ & $p$ & OR & Wald $\chi^{2}$ & $95 \% \mathrm{Cl}$ & $p$ \\
\hline Age & 1.1 & 11.953 & $1.1-1.1$ & 0.001 & 1.1 & 7.256 & $1.0-1.1$ & 0.007 \\
\hline Female gender & 0.5 & 4.661 & $0.2-0.9$ & 0.031 & 0.5 & 1.796 & $0.2-1.4$ & 0.180 \\
\hline Living alone & 0.5 & 7.550 & $0.3-0.8$ & 0.006 & 0.6 & 2.194 & $0.3-1.2$ & 0.139 \\
\hline Mild depressive symptoms & 1.0 & 0.002 & $0.6-1.7$ & 0.968 & 1.6 & 1.743 & $0.8-3.3$ & 0.187 \\
\hline Moderate to severe depressive symptoms & 4.6 & 18.745 & $2.3-9.1$ & $<0.0001$ & 3.2 & 6.611 & $1.3-7.8$ & 0.010 \\
\hline Mini Mental State Examination & 0.9 & 14.198 & $0.8-0.9$ & $<0.0001$ & 1.0 & 0.308 & $0.9-1.1$ & 0.579 \\
\hline Charlson Comorbidity Index & 1.4 & 13.254 & $1.2-1.6$ & $<0.0001$ & 1.2 & 3.753 & $1.0-1.6$ & 0.053 \\
\hline Albumin serum levels & 0.4 & 11.559 & $0.2-0.8$ & 0.016 & 0.3 & 5.404 & $0.1-0.8$ & 0.020 \\
\hline Walking ability before fracture ${ }^{a}$ & 0.8 & 25.339 & $0.7-0.8$ & $<0.0001$ & 0.8 & 8.510 & $0.7-0.9$ & 0.004 \\
\hline Number of drugs & 1.2 & 5.842 & $1.1-1.4$ & 0.001 & 1.1 & 3.132 & $1.0-1.3$ & 0.077 \\
\hline Antidepressants & 2.1 & 4.861 & $1.1-4.2$ & 0.027 & 1.8 & 2.179 & $0.8-4.2$ & 0.140 \\
\hline
\end{tabular}

Walking dependence at discharge was defined as a score $\geq 12 / 15$ in the corresponding item of Barthel Index. Mild depressive symptoms indicates scores between 6 and 9 at the Geriatric Depression Scale; Moderate to severe depressive symptoms indicates scores between 10 and 15 at the Geriatric Depression Scale. ${ }^{a}$ walking ability refers to the corresponding sub-item of the Barthel Index OR denotes Odds Ratio, Wald $\chi^{2}$ denoted Wald $\chi^{2}$, 95\% CI denotes Confidence Intervals, and $p$-value the associated significance computed in unadjusted and adjusted regression models. Variables predicting functional recovery were entered in a multivariate regression models as follows: age, Mini Mental State Examination, Charlson comorbidity index, albumin serum levels, walking sub-item before fracture, number of drugs and antidepressants were entered as continuous variables while gender, living alone, mild depressive symptoms, moderate to severe depressive symptoms as dichotomous.

In order to assess the power of depressive symptoms and other variables to predict failure to recover walking independence at discharge and the occurrence of 1year adverse outcomes, two multiple stepwise regressions were created. In the first one (Table 2), variables independently associated with failing the recovery of walking independence at discharge were age (odds ratio, O.R $=1.1 ; 95 \%$ confidence interval, $\mathrm{CI}=1.0$
$1.1 ; p=0.007)$, moderate to severe depressive symptoms $(\mathrm{O} . \mathrm{R}=3.2 ; 95 \% \mathrm{CI}=1.3-7.8 ; p=0.010)$, albumin serum levels $(\mathrm{O} . \mathrm{R}=0.3 ; 95 \% \mathrm{CI}=0.1-0.8 ; p=0.20)$ and walking ability before fracture $(\mathrm{O} . \mathrm{R}=0.8 ; 95 \%$ $\mathrm{CI}=0.7-0.9 ; \quad p=0.001)$. In the second analysis (Table 3), variables independently associated with 1 -year adverse events were age $(\mathrm{O} . \mathrm{R}=1.1 ; 95 \% \mathrm{CI}=1.0$ $1.1 ; p=0.011)$, moderate to severe depressive symptoms

Table 3 Independent predictors of adverse events at 1 year in 280 patients consecutively admitted to a Department of Rehabilitation and Aged Care Unit after hip fracture surgery in unadjusted and adjusted multiple logistic regression models

\begin{tabular}{|c|c|c|c|c|c|c|c|c|}
\hline & \multicolumn{8}{|c|}{ Adverse events at 1 year } \\
\hline & \multicolumn{4}{|c|}{ Unadjusted } & \multicolumn{4}{|c|}{ Adjusted } \\
\hline & OR & Wald $\chi^{2}$ & $95 \% \mathrm{Cl}$ & $p$ & OR & Wald $\chi^{2}$ & $95 \% \mathrm{Cl}$ & $p$ \\
\hline Age & 1.1 & 13.808 & $1.1-1.2$ & $<0.0001$ & 1.1 & 6.464 & $1.0-1,1$ & 0.011 \\
\hline Female gender & 0.6 & 1.179 & $0.3-1.4$ & 0.278 & 0.5 & 1.640 & $0.2-1.4$ & 0.200 \\
\hline Mild depressive symptoms & 1.0 & 0.009 & $0.5-2.0$ & 0.924 & 2.1 & 3.010 & $0.9-4.9$ & 0.083 \\
\hline Moderate to severe depressive symptoms & 5.0 & 20.089 & $2.5-10.2$ & $<0.0001$ & 3.6 & 7.331 & $1.4-9.1$ & 0.007 \\
\hline Mini Mental State Examination & 0.9 & 12.413 & $0.4-1.9$ & $<0.0001$ & 0.9 & 2.120 & $0.9-1.0$ & 0.145 \\
\hline Albumin serum levels & 0.4 & 3.539 & $0.2-1.0$ & 0.060 & 0.3 & 4.584 & $0.1-0.9$ & 0.032 \\
\hline Failure to recover walking independence at Discharge & 4.9 & 22.808 & $2.6-9.5$ & $<0.0001$ & 2.5 & 5.659 & $1.2-5.2$ & 0.017 \\
\hline Number of drugs & 1.1 & 3.082 & $1.0-1.3$ & 0.079 & 1.0 & 1.194 & $0.9-1.2$ & 0.784 \\
\hline Antidepressants & 1.5 & 1.471 & $0.8-2.9$ & 0.225 & 1.8 & 2.079 & $0.8-4.0$ & 0.149 \\
\hline
\end{tabular}

Adverse outcome included 1 year - death or institutionalization. Mild depressive symptoms indicates scores between 6 and 9 at the Geriatric Depression Scale; Moderate to severe depressive symptoms indicates scores between 10 and 15 at the Geriatric Depression Scale.

OR denotes Odds Ratio, denoted Wald $\chi^{2}, 95 \%$ CI denotes Confidence Intervals and $p$-value the associated significance computed in unadjusted and adjusted regression models.

Variables predicting 1-year adverse outcomes were entered in a multivariate regression models as follows: age, Mini Mental State Examination, albumin serum levels, number of drugs and antidepressants were entered as continuous variables while gender, mild depressive symptoms, moderate to severe depressive symptoms, failure to recover walking independence at discharge as dichotomous. 
(O.R $=3.6 ; 95 \% \mathrm{CI}=1.4-9.1 ; p=0.007)$, failure to recover walking independence at discharge $(\mathrm{O} . \mathrm{R}=2.5$; $95 \% \mathrm{CI}=1.2-5.2 ; p=0.017)$ and albumin serum levels $(\mathrm{O} . \mathrm{R}=0.3 ; 95 \% \mathrm{CI}=0.1-0.9 ; p=0.032)$.

Moderate to severe depressive symptoms were significantly correlated (Pearson's correlations) both with failure to recover walking dependence at discharge $(p=0.000)$ and 1 year adverse events $(p=0.000)$. In the logistic regression models, depressive symptoms predicted both failure to recover walking dependence at discharge $(\beta=0.35)$ and 1 year adverse events $(\beta=0.20)$; failure to recover walking dependence predicted 1 year adverse events $(\beta=0.20)$. The value of Sobel test statistic for the mediation of failing walking independence at discharge was 3.200922, yielding a probability $=0.000685$ (one-tailed) and $=0.001370$ (two-tailed), respectively.

\section{Discussion}

Our study shows that moderate to severe depressive symptoms predict failure to recover walking independence at discharge and adverse clinical outcomes at 1 year (i.e. death or institutionalization) in post-surgery HF rehabilitation. Mild depressive symptoms do not have a significant predictive power.

The negative impact of depressive symptoms on rehabilitation has been previously detected by Lenze et al. (2004), who found that depression is associated to poor participation in rehabilitation program which in turn can affect functional outcomes. Depression can reduce motivation, generating apathy, low energy and pessimism (American Psychiatric Association, 1994). In this context, patients may have a difficulty in deriving benefit from rehabilitation which can result in unfavourable outcomes, such as prolonged length of stay or, paradoxically, a premature discharge (Gantner et al., 2003). Moreover, depressive symptoms may influence physical therapists in empathizing with their patients, thus failing to approach them with the appropriate intensity of treatment (Bellelli and Trabucchi, 2009). However, not all studies confirmed the association. For example, a recent study by Arinzon et al. (2009) found that recovery post-HF depends largely on patient's pre-fracture health and functional ability, but not on affective status.

The lack of unequivocal data about the relationship between functional recovery and depressive symptoms may be related to the variability among studies in the methods used to assess depression or depressive symptoms. In line with this interpretation, it should be noted that some studies defined depression using categorical criteria (Mossey et al., 1989; Hershkovitz et al., 2007), while others used screening tools such as the Hamilton Depression Scale (HAM-D) (Lenze et al., 2004; Lenze et al., 2007), the Center of Epidemiological Study-Depression (CES-D) (Magaziner et al., 2000; Haentjens et al., 2005) and the GDS (Lieberman et al., 2006; Givens et al., 2008). Moreover, studies differed also for the cut-off chosen to detect the presence of depressive symptoms, significantly affecting the relationship of this condition with the functional outcome. For example, in two studies that fixed the cut-off of the GDS at 10/30 (Lieberman et al., 2006) and of the HAM-D at 10/17 (Lenze et al., 2007), the authors failed to find a significant association, while in other two studies that used a higher cut-off or defined depression according to a clinical classification (Lenze et al., 2004; Hershkovitz et al., 2007), this association became evident. The results of our study are in line with this assumption and also with a recent study by Chiang, finding that the 15-items GDS is more accurate to detect moderate or severe levels of depression than mild ones (Chiang et al., 2009).

Another possible explanation of discrepancies among studies may be related to the methods used to assess the functional outcome. In fact, despite few exceptions (Gindin et al., 2007; Fusco et al., 2009), the majority of previous studies evaluated it with measures of patient's global functioning, such as Functional Independence Measure, BI and autonomy in Activity of Daily Living (Fredman et al., 2006; Lieberman et al., 2006; Hershkovitz et al., 2007; Lenze et al., 2007; Givens et al., 2008; Arinzon et al., 2010) or with measures of performance such as gait speed (Magaziner et al., 2000). However, these tools may underestimate the effect of depressive symptoms on functional outcome. In fact, as it has been previously shown (Magaziner et al., 2000), the recuperation trajectory following HF rehabilitation occurs with a typical sequence that starts with the recovery of impairments, follows with the recovery of functional limitations (such as, for example, neuromuscular functions), and then with the recovery of lower extremity functions. The recuperation of global function in Activities of Daily Living (ADL) and of gait speed obviously requires periods of time longer than those usually provided in a post-acute care rehabilitative settings. In this context, a strength of our study is that we used an outcome measure which can be considered a potentially achievable outcome for all post-surgical HF undergoing a rehabilitative training. A second strength is that we selected only patients who were completely unable to walk on admission, thus reducing the risk of misinterpretation of motor recovery at discharge. 
With regard to 1-year adverse events, several studies (Holmes and House, 2000; Nightingale et al., 2001; Dalle Carbonare et al., 2009) have shown that depressive symptoms are associated with increased morbidity and may negatively affect health status when associated with chronic diseases (Dalle Carbonare et al., 2009). This could be due to sub-optimal disease control caused by poor self-care strategies, low adherence to medications and higher risk of drugs' adverse reactions (DiMatteo et al., 2000; Onder et al., 2003). Hence, it could be hypothesised that depression and depressive symptoms increases patients' susceptibility towards adverse events. An alternative explanation is that severe depressive symptoms may reflect a condition of frailty which requires time to express its adverse effects (Rozzini and Trabucchi, 2003).

A relevant finding of our study concerns the relationship among moderate to severe depressive symptoms, walking ability at discharge and 1-year adverse outcomes. We found that failure to recover walking independence at discharge mediates the relationship between moderate to severe depressive symptoms and the long-term outcomes, suggesting that depressed patients have an increased 1-year risk of death or institutionalization partly because they do not recover their walking ability at discharge from rehabilitation. This finding may have at least one possible implication. It should be hypothesized that patients with both moderate to severe depressive symptoms and unable to walk independently after HF rehabilitation might prolong their length of stay and intensify the physiotherapic regimen in order to increase the probability to recover walking. Another possibility is that more depressed patients should receive a more intensive antidepressant treatment, including drugs and psychological support.

Some limitations should be highlighted. First, the only measure to assess depressive symptoms was the 15-item GDS. However, previous studies showed that this is a reliable screening instrument for major depression according to IDC-10 and DSM-IV (Almeida and Almeida, 1999). Second, the facts that our study was conducted in a single site and that we are not able to know if patients lost to follow-up at 1 year have been institutionalized in the meanwhile may limit the generalizability of our result. However, it should be remarked that the rate of 1-year drop-out found in our study is in line with, or even lower than those of previous studies on similar population (Magaziner et al., 2000; Shyu et al., 2004). Finally, variables with a possible influence on functional recovery, such as fracture site, type of anaesthesia, degree of preoperative risk and levels of Vitamin D were not assessed in this study.
Key points

- The impact of depressive symptoms in post-HF surgery rehabilitation has been repeatedly evaluated with regard to patients' functional recovery at discharge and other adverse outcomes, but with conflicting results.

- Previous studies assessed the effect of presence of depressive symptoms irrespectively of their severity and did not evaluated functional recovery in terms of walking independence at discharge, although this represents the main goal of HF rehabilitation.

- Our study found that only moderate to severe depressive symptoms (GDS $\geq 10 / 15$ ) affect the recovery of walking independence after HF rehabilitation and that they are also associated with severe adverse events at 1 year. The failure to recover walking independence at discharge partially mediates the relationship between moderate to severe depressive symptoms and 1-year adverse events.

\section{Conclusion}

In conclusion, our study shows that moderate to severe depressive symptoms in post-HF surgery patients, as measured by the GDS, are associated to poor walking recovery after rehabilitation and to an increased risk of the institutionalization or death at 1 year. In addition to those of previous studies, these results may provide valuable clues for designing trials of interventions and developing individualized rehabilitation pathways that consider moderate to severe affective disorders as a specific target of interest.

\section{Conflict of interest}

None declared.

\section{REFERENCES}

Almeida OP, Almeida SA. 1999. Short versions of the geriatric depression scale: a study of their validity for the diagnosis of a major depressive episode according to ICD-10 and DSM-IV. Int J Geriatr Psychiatry 14: 858-865.

American Psychiatric Association. 1994. Diagnostic and Statistical Manual of Mental Disorders, 4th edition. American Psychiatric Association Washington (DC).

Arinzon Z, Shabat S, Peisakh A, et al. 2010. Gender differences influence the outcome of geriatric rehabilitation following hip fracture. Arch Gerontol Geriatr 50: 86-91.

Bellelli G, Frisoni GB, Turco R, et al. 2008. Depressive symptoms combined with dementia affect 12-months survival in elderly patients after rehabilitation post-hip fracture surgery. Int J Geriatr Psychiatry 23: 1073-1077. 
Bellelli G, Trabucchi M (eds). 2009. Riabilitare l'anziano: basi culturali e strumenti di lavoro. Carocci Faber: Torino.

Brink TL, Yesavage JA, Lum O, et al. 1982. Screening tests for geriatric depression. Clin Gerontol 1: 37-43.

Charlson ME, Pompei P, Ales KL, et al. 1987. A new method of classifying prognostic comorbidity in longitudinal studies: development and validation. J Chronic Dis 40: 373-383.

Chiang KS, Green K, Cox EO. 2009. Rasch analysis of the Geriatric Depression Scaleshort form. Gerontologist 49: 262-275.

Crotty M, Unroe K, Cameron ID, et al. 2009. Rehabilitation interventions for improving physical and psychosocial functioning after hip fracture. Cochrane Batabase Syst Rev 1: CD007624.

Cullum S, Metcalfe C, Todd C, et al. 2008. Does depression predict adverse outcomes for older medical inpatients? A prospective cohort study of individuals screened for a trial. Age Ageing 37: 690-695.

Dalle Carbonare L, Maggi S, Noale M, et al. 2009. Physical disability and depressive symptomatology in an elderly population: a complex relationship. The Italian Longitudinal Study on Ageing (ILSA). Am J Geriatr Psychiatry 17: 144-154.

de Luise C, Brimacombe M, Pedersen L, et al. 2008. Comorbidity and mortality following hip fracture: a population-based cohort study. Aging Clin Exp Res 20: 412-418.

DiMatteo MR, Lepper HS, Croghan TW. 2000. Depression is a risk factor for noncompliance with medical treatment: meta-analysis of the effects of anxiety and depression on patient adherence. Arch Intern Med 160: 2101-2107.

Folstein MF, Folstein SE, McHugh PR. 1975. Mini Mental State. A practical method for grading the cognitive state of patients for the clinician. J Psychiatr Res 12: 189 198.

Fredman L, Hawkes WG, Black S, et al. 2006. Elderly patients with hip fracture with positive affect have better functional recovery over 2 years. J Am Geriatr Soc 54: 1074-1081.

Fusco D, Bochicchio GB, Onder G, et al. 2009. Predictors of rehabilitation outcome among frail elderly patients living in the community. J Am Med Dir Assoc 10(5): $335-341$.

Gantner AB, Schubert DS, Wolf SR, et al. 2003. Screening for depression in a geriatric rehabilitation sample. Int J Psychiatry Med 33: 333-341.

Gindin J, Walter-Ginzburg A, Geitzen M, et al. 2007. Predictors of rehabilitation outcomes: a comparison of Israeli and Italian geriatric post-acute care (PAC) facilities using the minimum data set (MDS). J Am Med Dir Assoc 8: 233-242.
Givens JL, Sanft TB, Marcantonio ER. 2008. Functional recovery after hip fracture: the combined effects of depressive symptoms, cognitive impairment, and delirium. $J$ Am Geriatr Soc 56: 1075-1079.

Haentjens P, Autier P, Barette M, et al. 2005. Predictors of functional outcome following intracapsular hip fracture in elderly women. A one-year prospective cohort study. Injury 36: 842-850.

Hershkovitz A, Kalandariov Z, Hermush V, et al. 2007. Factors affecting short-term rehabilitation outcomes of disabled elderly patients with proximal hip fracture. Arch Phys Med Rehabil 88: 916-921.

Holmes J, House A. 2000. Pychiatric illness in hip fracture. Age Ageing 29: 537-546. Laforgia R, Maggi S, Marzari C, et al. 2006. Epidemiology of femoral neck fracture in old people in Italy. J Bone Joint Surg Br 88B(Suppl 1): 42.

Lenze EJ, Munin MC, Dew MA, et al. 2004. Adverse effects of depression and cognitive impairment on rehabilitation participation and recovery from hip fracture. Int J Geriatr Psychiatry 19: 472-478.

Lenze EJ, Skidmore ER, Dew MA, et al. 2007. Does depression, apathy, or cognitive impairment reduce the benefit of inpatient rehabilitation facilities for elderly hip fracture patients? Gen Hosp Psychiatry 29: 141-146.

Lieberman D, Friger M, Lieberman D. 2006. Inpatient rehabilitation outcome after hip fracture surgery in elderly patients: a prospective cohort study of 946 patients. Arch Phys Med Rehabil 87: 167-171.

Magaziner J, Hawkes W, Hebel JR. 2000. Recovery from hip fracture in eight areas of function. J Gerontol A Biol Sci Med Sci 55: M498-M507.

Mossey JM, Mutran E, Knott K, et al. 1989. Determinants of recovery 12 months after hip fracture: the importance of psychosocial factors. Am J Public Health 79: 279286.

Nightingale S, Holmes J, Mason J, et al. 2001. Psychiatric illness and mortality after hip fracture. Lancet 357: 1264-1265.

Onder G, Penninx B, Landi F, et al. 2003. Depression and adverse drug reactions among hospitalized older adults. Arch Intern Med 163: 301-305.

Rozzini R., Trabucchi M. 2003. Depression and negative outcomes in patients with heart failure. Arch Intern Med 163: 498.

Shah S, Vanclay F, Cooper B. 1989. Improving the sensitivity of the Barthel Index for stroke rehabilitation. J Clin Epidemiol 42: 703-709.

Shyu YIL, Chen MC, Liang J, et al. 2004. Predictors of functional recovery for hip fractured elders during 12 months following hospital discharge: a prospective study on a Taiwanese sample. Osteoporos Int 15: 475-482.

Yesavage JA, Brink TL, Rose TL, et al. 1983. Development and validation of a geriatric depression screening scale: a preliminary report. J Psychiatr Res 17: 37-49. 